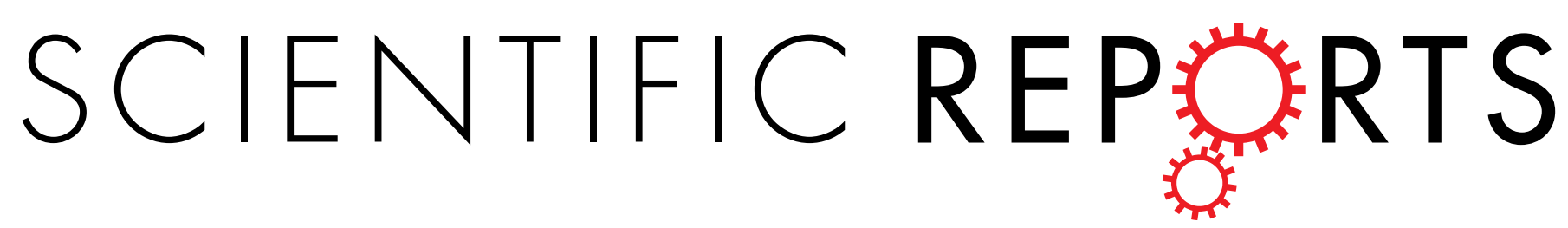

\title{
OPEN Author Correction: A Donor Quality Index for liver transplantation: development, internal and external validation
}

\author{
Audrey Winter ${ }^{1,2,3}$, Cyrille Féray ${ }^{4}$, Etienne Audureau ${ }^{5}$, Daniel Azoulay ${ }^{6}$, Corinne Antoine ${ }^{7}$, \\ Jean-Pierre Daurès ${ }^{1,2} \&$ Paul Landais $^{1}$ \\ Correction to: Scientific Reports https://doi.org/10.1038/s41598-018-27960-7, published online 29 June 2018 \\ This Article contains errors.
}

In Table 1, the formatting for the header "Recipient characteristics" is incorrect. The correct Table 1 appears below:
${ }^{1}$ Department of Biostatistics, UPRES EA2415, Clinical Research University Institute, Montpellier, France. ${ }^{2}$ Beau Soleil Clinic, Montpellier, France. ${ }^{3}$ Department of Radiological Sciences, University of California, Los Angeles, CA, USA. ${ }^{4}$ Department of Hepatology, Henri Mondor Hospital, Créteil, France. ${ }^{5}$ Department of Public Health, Henri Mondor Hospital, Créteil, France. ${ }^{6}$ Department of Surgery, Henri Mondor Hospital, Créteil, France. ${ }^{7}$ Agence de la Biomédecine, Saint-Denis, France. Correspondence and requests for materials should be addressed to A.W. (email: audrey.winter89@gmail.com) 


\begin{tabular}{|c|c|c|c|}
\hline Donor characteristics & & Mean survival (sd) & P-value \\
\hline Sex (\%): & & & 0.37 \\
\hline Male & 55.01 & $4.55(0.05)$ & \\
\hline Female & 44.99 & $4.56(0.08)$ & \\
\hline Cause of death (\%): & & & $<0.01$ \\
\hline Cerebrovascular accident & 60.29 & $4.44(0.05)$ & \\
\hline Trauma & 24.64 & $4.69(0.07)$ & \\
\hline Anoxia & 12.02 & $4.80(0.11)$ & \\
\hline Other & 3.05 & $4.72(0.21)$ & \\
\hline Diabetes (\%): & & & 0.98 \\
\hline yes & 7.75 & $4.56(0.14)$ & \\
\hline no & 92.25 & $4.56(0.04)$ & \\
\hline $\mathrm{ABO}$ group (\%): & & & 0.22 \\
\hline $\mathrm{A}$ & 44.58 & $4.57(0.06)$ & \\
\hline $\mathrm{B}$ & 9.87 & $4.72(0.12)$ & \\
\hline $\mathrm{O}$ & 41.93 & $4.49(0.06)$ & \\
\hline $\mathrm{AB}$ & 3.61 & $4.78(0.19)$ & \\
\hline Hypertension (\%): & & & 0.21 \\
\hline yes & 35.70 & $4.49(0.07)$ & \\
\hline no & 64.30 & $4.60(0.05)$ & \\
\hline Malignancy (\%): & & & 0.43 \\
\hline yes & 1.89 & $4.72(0.26)$ & \\
\hline no & 98.11 & $4.49(0.04)$ & \\
\hline Alcohol (\%): & & & 0.53 \\
\hline yes & 15.02 & $4.50(0.10)$ & \\
\hline no & 84.98 & $4.57(0.04)$ & \\
\hline Smoking (\%): & & & 0.47 \\
\hline yes & 37.59 & $4.59(0.06)$ & \\
\hline no & 62.41 & $4.53(0.05)$ & \\
\hline Drugs (\%): & & & 0.52 \\
\hline yes & 4.17 & $4.65(0.19)$ & \\
\hline no & 95.83 & $4.56(0.04)$ & \\
\hline Hepatitis C virus antibody (\%): & & & 0.46 \\
\hline+ & 0.45 & $4.09(0.56)$ & \\
\hline- & 99.55 & $4.53(0.04)$ & \\
\hline Hepatitis B core antibody (\%): & & & 0.62 \\
\hline+ & 4.39 & $4.61(0.18)$ & \\
\hline- & 95.61 & $4.53(0.04)$ & \\
\hline Inotropes (dobutamine, dopamine, noradrenaline, epinephrine) (\%): & & & 0.72 \\
\hline yes & 39.16 & $4.57(0.06)$ & \\
\hline no & 60.84 & $4.55(0.05)$ & \\
\hline Liver type (\%): & & & 0.44 \\
\hline Partial/split & 5.10 & $4.43(0.17)$ & \\
\hline Total & 94.90 & $4.55(0.04)$ & \\
\hline Age (\%): & & & $<0.001$ \\
\hline$\leq 69$ & 76.47 & $4.64(0.04)$ & \\
\hline$>69$ & 23.53 & $4.27(0.09)$ & \\
\hline Height (\%): & & & 0.07 \\
\hline$<162$ & 22.62 & $4.42(0.08)$ & \\
\hline$\geq 162$ & 77.38 & $4.60(0.04)$ & \\
\hline Weight, mean (sd) & $72.88(15.17)$ & & \\
\hline BMI, mean (sd) & $25.33(4.62)$ & & \\
\hline Sodium: latest (mmol/L) (\%): & & & 0.04 \\
\hline $136-146$ & 43.85 & $4.47(0.06)$ & \\
\hline other & 56.15 & $4.63(0.05)$ & \\
\hline Sodium: highest (mmol/L), median (range) & $149(120-180)$ & & \\
\hline MDRD creatinine clearance: latest $\left(\mathrm{ml} / \mathrm{min} / 1.73 \mathrm{~m}^{2}\right)(\%)$ : & & & 0.01 \\
\hline$<60$ & 25.30 & $4.3850 .08)$ & \\
\hline \multicolumn{4}{|l|}{ Continued } \\
\hline
\end{tabular}




\begin{tabular}{|c|c|c|c|}
\hline Donor characteristics & & Mean survival (sd) & P-value \\
\hline $60-89$ & 29.51 & $4.55(0.07)$ & \\
\hline$\geq 90$ & 45.19 & $4.66(0.06)$ & \\
\hline MDRD creatinine clearance: lowest $\left(\mathrm{ml} / \mathrm{min} / 1.73 \mathrm{~m}^{2}\right)(\%):$ & & & 0.01 \\
\hline$<60$ & 35.35 & $4.41(0.07)$ & \\
\hline $60-89$ & 36.83 & $4.61(0.06)$ & \\
\hline$\geq 90$ & 27.82 & $4.68(0.07)$ & \\
\hline Aspartate aminotransferase: latest (U/L), median (range) & $39(0-2000)$ & & \\
\hline Aspartate aminotransferase: highest (U/L), median (range) & $51(9-2000)$ & & \\
\hline Alanine transaminase: latest $(\mathrm{U} / \mathrm{L})(\%)$ : & & & 0.04 \\
\hline $7-41$ & 67.33 & $4.50(0.05)$ & \\
\hline other & 32.67 & $4.67(0.07)$ & \\
\hline Alanine transaminase: highest (U/L), median (range) & $33(1-2000)$ & & \\
\hline Total bilirubin: latest ( $\mu \mathrm{mol} / \mathrm{L})$, median (range) & $10(0-150)$ & & \\
\hline Total bilirubin: highest ( $\mu \mathrm{mol} / \mathrm{L})$, median (range) & $12(1-150)$ & & \\
\hline Alkaline phosphatases: latest (U/L) (\%): & & & 0.06 \\
\hline$<33$ & 4.44 & $4.26(0.19)$ & \\
\hline $33-96$ & 76.12 & $4.54(0.04)$ & \\
\hline$>96$ & 19.44 & $4.71(0.08)$ & \\
\hline Alkaline phosphatases: highest (U/L) (\%): & & & 0.09 \\
\hline$<33$ & 1.78 & $4.22(0.31)$ & \\
\hline $33-96$ & 72.22 & $4.51(0.05)$ & \\
\hline$>96$ & 26.00 & $4.67(0.07)$ & \\
\hline Gamma glutamyl transpeptidase: latest (U/L), median (range) & $30(0-1477)$ & & \\
\hline Gamma glutamyl transpeptidase: highest (U/L), median (range) & $36(1-1835)$ & & \\
\hline Intensive care unit stay (in days) (\%): & & & $<0.001$ \\
\hline$\leq 4$ & 79.68 & $4.48(0.04)$ & \\
\hline$>4$ & 29.32 & $4.83(0.08)$ & \\
\hline Estimated distance between donor and recipient location (minutes) (\%): & & & $<0.01$ \\
\hline$<15$ & 22.60 & $4.77(0.08)$ & \\
\hline$\geq 15$ & 77.40 & $4.50(0.04)$ & \\
\hline "Hors tour" (\%): & & & 0.84 \\
\hline yes & 6.08 & $4.57(0.16)$ & \\
\hline no & 93.92 & $4.55(0.04)$ & \\
\hline Donor risk index, mean (sd) & $1.65(0.40)$ & - & - \\
\hline Eurotransplant donor risk index, mean (sd) & $1.63(0.36)$ & - & - \\
\hline UK Donor Liver Index, mean (sd) & $1.15(0.26)$ & - & - \\
\hline Recipient characteristics & & Mean survival (sd) & P-value \\
\hline Sex $(\%):$ & & & 0.91 \\
\hline Male & 73.54 & $4.55(0.05)$ & \\
\hline Female & 26.46 & $4.56(0.08)$ & \\
\hline Cancer (\%): & & & 0.29 \\
\hline yes & 29.00 & $4.62(0.07)$ & \\
\hline no & 71.00 & $4.53(0.05)$ & \\
\hline Decompensated cirrhosis (\%): & & & $<0.001$ \\
\hline yes & 37.74 & $4.73(0.06)$ & \\
\hline no & 62.26 & $4.46(0.05)$ & \\
\hline Non-cirrhotic liver disease (\%): & & & 0.63 \\
\hline yes & 1.41 & $4.63(0.30)$ & \\
\hline no & 98.59 & $4.48(0.04)$ & \\
\hline Emergency (\%): & & & $<0.001$ \\
\hline yes & 6.72 & $3.78(0.17)$ & \\
\hline no & 93.28 & $4.58(0.04)$ & \\
\hline MELD exception (\%): & & & 0.36 \\
\hline yes & 18.00 & $4.45(0.09)$ & \\
\hline no & 82.00 & $4.57(0.04)$ & \\
\hline Previous transplantation (\%): & & & $<0.001$ \\
\hline yes & 8.58 & $3.76(0.15)$ & \\
\hline
\end{tabular}




\begin{tabular}{|c|c|c|c|}
\hline Recipient characteristics & & Mean survival (sd) & P-value \\
\hline no & 91.42 & $4.60(0.04)$ & \\
\hline On dialysis (\%): & & & $<0.001$ \\
\hline yes & 5.33 & $3.43(0.19)$ & \\
\hline no & 94.67 & $4.58(0.04)$ & \\
\hline Medical condition before LT (\%): & & & $<0.001$ \\
\hline Intensive care unit & 17.70 & $3.88(0.11)$ & \\
\hline Hospital (no intensive care unit) & 13.88 & $4.60(0.10)$ & \\
\hline Not hospitalized & 68.42 & $4.71(0.04)$ & \\
\hline Hepatitis B core antibody (\%): & & & 0.73 \\
\hline+ & 20.05 & $4.53(0.09)$ & \\
\hline- & 79.95 & $4.56(0.04)$ & \\
\hline Hepatitis C virus antibody (\%): & & & $<0.001$ \\
\hline+ & 23.83 & $4.23(0.08)$ & \\
\hline- & 76.17 & $4.66(0.04)$ & \\
\hline Diabetes (\%): & & & 0.06 \\
\hline yes & 22.67 & $4.42(0.08)$ & \\
\hline no & 77.33 & $4.60(0.04)$ & \\
\hline Encephalopathy (\%): & & & $<0.001$ \\
\hline grade 1 & 68.14 & $4.62(0.05)$ & \\
\hline grade 2 & 24.84 & $4.52(0.08)$ & \\
\hline grade 3 & 7.02 & $3.95(0.17)$ & \\
\hline ABO group (\%): & & & 0.80 \\
\hline A & 45.44 & $4.56(0.06)$ & \\
\hline $\mathrm{B}$ & 11.16 & $4.63(0.11)$ & \\
\hline $\mathrm{O}$ & 39.03 & $4.55(0.06)$ & \\
\hline $\mathrm{AB}$ & 4.37 & $4.43(0.19)$ & \\
\hline Age, mean (sd) & $53.24(10.39)$ & & \\
\hline Body mass index (\%): & & & 0.20 \\
\hline$<18.5$ & 3.56 & $4.35(0.21)$ & \\
\hline $18.5-25$ & 44.69 & $4.50(0.06)$ & \\
\hline$\geq 25$ & 51.75 & $4.62(0.05)$ & \\
\hline Model for end stage liver disease before LT (\%): & & & $<0.001$ \\
\hline$<29$ & 72.73 & $4.65(0.04)$ & \\
\hline$\geq 29$ & 27.27 & $4.32(0.08)$ & \\
\hline Waiting time (in days) (\%): & & & $<0.001$ \\
\hline$<21$ & 24.54 & $4.34(0.08)$ & \\
\hline$\geq 21$ & 75.46 & $4.63(0.04)$ & \\
\hline Follow-up (years), mean (sd) & $2.33(1.63)$ & & \\
\hline
\end{tabular}

Table 1. Donor and recipient characteristics with Log-rank test p-value for qualitative variables and quantitative variables across risk groups when significant threshold was met.

In addition, Table 2 contains formatting errors. The correct Table 2 appears below: 


\begin{tabular}{|c|c|c|c|c|c|}
\hline & Covariates & Model 1 & Model 2 & Model 3 & Final model \\
\hline \multirow{9}{*}{ Donor } & Age & $x$ & $\times$ & $x$ & $\times$ \\
\hline & Cause of death & $x$ & $\times$ & $\times$ & $\times$ \\
\hline & Intensive care unit stay & $x$ & $\times$ & $\times$ & $\times$ \\
\hline & $\mathrm{ABO}$ group & $x$ & & & $\times$ (adjustment) \\
\hline & MDRD creatinine clearance: lowest & $x$ & & & $x$ \\
\hline & Partial/split liver & $x$ & & & $x$ \\
\hline & Alcohol & $x$ & & & \\
\hline & Alkanine phosphatases: latest & & $\times$ & & \\
\hline & Estimated distance & & & $\times$ & \\
\hline \multirow{8}{*}{ Recipient } & Re-transplantation & $\times$ & $\times$ & $\times$ & $x$ \\
\hline & On dialysis & $x$ & $\times$ & $\times$ & $x$ \\
\hline & Medical condition before LT & $\times$ & $\times$ & $\times$ & $\times$ \\
\hline & Hepatitis C virus antibody & $x$ & $\times$ & $x$ & $x$ \\
\hline & Diabetes & $x$ & $x$ & $x$ & $x$ \\
\hline & Decompensated cirrhosis & $x$ & $\times$ & $x$ & $x$ \\
\hline & MELD exception & $\times$ & & & $\times$ \\
\hline & ABO group & $\times$ & & & $x$ \\
\hline \multirow{3}{*}{ Perfomances } & C-index & $0.626(0.009)$ & $0.616(0.009)$ & $0.616(0.009)$ & $0.625(0.009)$ \\
\hline & K statistic & $0.617(0.007)$ & $0.611(0.008)$ & $0.610(0.008)$ & $0.616(0.007)$ \\
\hline & $R_{D}^{2}$ & $0.518(0.020)$ & $0.492(0.021)$ & $0.492(0.021)$ & $0.516(0.021)$ \\
\hline
\end{tabular}

Table 2. Retained covariates in the different selection way. Covariates in bold are those which appeared in the three selection models. Model 1: A complete model with selection according to the Akaiké criterion; Model 2: (1) log rank tests with a threshold of $20 \%$, (2) multivariate model included the selected covariates with a selection threshold set at 20\%; Model 3: (1) log rank tests with a threshold of 20\%, (2) two multivariate models for donor and recipient with the selected covariates with a selection threshold set at $20 \%$, (3) multivariate model with all the covariates selected with a selection threshold set at $20 \%$. The retained full model included all the variables present in at least two of the three models and the set of covariates retained in addition.

Finally, there are errors in Table 5, where the formatting of the "Donor characteristics" header is incorrect. Additionally, the word "Hospital" is incorrectly given as "Hosptial" and the P-values for "ICU stay (in days)" and "Clearance MDRD: lowest $(\mathrm{ml} / \mathrm{min} / 1.73 \mathrm{~m} 2)$ " are omitted. The correct Table 5 appears below as Table 3: 


\begin{tabular}{|c|c|c|c|}
\hline Recipient characteristics & $2009-2013(n=3961)$ & $2014(n=1048)$ & P-values \\
\hline Expert component (no) & $3248(82 \%)$ & $874(83.4 \%)$ & 0.31 \\
\hline $\mathrm{ABO}$ group & & & 0.59 \\
\hline $\mathrm{A}$ & $1800(45.44 \%)$ & $465(44.37 \%)$ & \\
\hline $\mathrm{AB}$ & $173(4.37 \%)$ & $38(3.63 \%)$ & \\
\hline $\mathrm{B}$ & $442(11.16 \%)$ & $118(11.26 \%)$ & \\
\hline $\mathrm{O}$ & $1546(39.03 \%)$ & $427(40.74 \%)$ & \\
\hline Re-transplantation (no) & $3621(91.42 \%)$ & $961(91.7 \%)$ & 0.82 \\
\hline On dialysis (no) & $3750(94.67 \%)$ & $987(94.18 \%)$ & 0.58 \\
\hline Medical condition before LT & & & 0.45 \\
\hline Home & $2710(68.42 \%)$ & $705(67.27 \%)$ & \\
\hline Hospital & $550(13.89 \%)$ & $140(13.36 \%)$ & \\
\hline ICU & $701(17.7 \%)$ & $203(19.37 \%)$ & \\
\hline Hepatitis $\mathrm{C}$ virus antibody $(-)$ & $3017(76.17 \%)$ & $811(77.39 \%)$ & 0.43 \\
\hline Diabetes (no) & $3063(77.33 \%)$ & $793(75.67 \%)$ & 0.27 \\
\hline Decompensated cirrhosis (no) & $2466(62.26 \%)$ & $667(63.65 \%)$ & 0.43 \\
\hline Donor characteristics & $2009-2013(n=3961)$ & $2014(n=1048)$ & P-values \\
\hline $\mathrm{ABO}$ group & & & 0.75 \\
\hline $\mathrm{A}$ & $1766(44.58 \%)$ & $460(43.89 \%)$ & \\
\hline $\mathrm{AB}$ & $143(3.61 \%)$ & $32(3.05 \%)$ & \\
\hline B & $391(9.87 \%)$ & $102(9.73 \%)$ & \\
\hline $\mathrm{O}$ & $1661(41.93 \%)$ & $454(43.32 \%)$ & \\
\hline Age & & & $<0.01$ \\
\hline$\leq 69$ & \begin{tabular}{|l}
$3029(76.47 \%)$ \\
\end{tabular} & $710(67.75 \%)$ & \\
\hline$>69$ & $932(23.53 \%)$ & $338(32.25 \%)$ & \\
\hline $\mathrm{COD}$ & & & $<0.01$ \\
\hline Anoxia & $476(12.02 \%)$ & $150(14.31 \%)$ & \\
\hline CVA & $2388(60.29 \%)$ & $651(62.12 \%)$ & \\
\hline Trauma & $976(24.64 \%)$ & $231(22.04 \%)$ & \\
\hline Other & $121(3.05 \%)$ & $16(1.53 \%)$ & \\
\hline ICU stay (in days) & & & 0.23 \\
\hline$\leq 4$ & $3156(79.68 \%)$ & $853(81.39 \%)$ & \\
\hline$>4$ & $805(20.32 \%)$ & $195(18.61 \%)$ & \\
\hline $\begin{array}{l}\text { Clearance MDRD: lowest (ml/ } \\
\mathrm{min} / 1.73 \mathrm{~m}^{2} \text { ) }\end{array}$ & & & 0.55 \\
\hline$<60$ & $1400(35.34 \%)$ & $385(36.74 \%)$ & \\
\hline $60-89$ & $1459(36.83 \%)$ & $388(37.02 \%)$ & \\
\hline$\geq 90$ & $1102(27.82 \%)$ & $275(26.24 \%)$ & \\
\hline Liver type (entire) & $3759(94.9 \%)$ & $1007(96.09 \%)$ & 0.13 \\
\hline
\end{tabular}

Table 3. Differences in donor and recipient characteristics between the derivation and validation datasets (P-values, $\chi^{2}$ tests and ANOVA when appropriate).

Open Access This article is licensed under a Creative Commons Attribution 4.0 International License, which permits use, sharing, adaptation, distribution and reproduction in any medium or format, as long as you give appropriate credit to the original author(s) and the source, provide a link to the Creative Commons license, and indicate if changes were made. The images or other third party material in this article are included in the article's Creative Commons license, unless indicated otherwise in a credit line to the material. If material is not included in the article's Creative Commons license and your intended use is not permitted by statutory regulation or exceeds the permitted use, you will need to obtain permission directly from the copyright holder. To view a copy of this license, visit http://creativecommons.org/licenses/by/4.0/.

(c) The Author(s) 2018 